\title{
RhoGDI2 up-regulates P-glycoprotein expression via Rac1 in gastric cancer cells
}

\author{
Zhong Zheng ${ }^{1}$, Bingya $\mathrm{Liu}^{2}$ and Xiaohua $\mathrm{Wu}^{1 *}$
}

\begin{abstract}
Multidrug resistance (MDR) is a major clinical obstacle in treatment of gastric cancer. Previously, using 2D electrophoresis-mass spectrometry, we identified RhoGDI2 as a contributor to 5-FU resistance in colon cancer cells, and also confer gastric cancer cells resistance to 5-FU. Here, we reported RhoGDI2 also induced MDR in gastric cancer cell line (MKN-45). To explore the underlining mechanism, we detected the mRNA, protein expression, activity of P-glycoprotein (P-gp) in MKN-45 stably transfected with RhoGDI2 expressing or control vector. All the mRNA, protein level, activity were increased by 130\%, 230\%, 35\% respectively after ectopic expression of RhoGDI2. RhoGDI2 was correlated with P-gp expression in gastric cancer tissues as detected by immunohistochemistry. To further study how RhoGDI2 up-regulates P-gp expression, we tested the activity of Rac1 in MKN-45/RhoGDI2 and MKN-45/GFP. Ectopic expression of RhoGDI2 increased Rac1 activity $(P<0.05)$. For more important, silencing of Rac1 expression by siRNA decreased P-gp expression to undetectable level. Overall, these findings suggest that RhoGDI2 up-regulates P-gp expression via Rac1 to induce MDR.
\end{abstract}

Keywords: RhoGDI2, Multidrug resistance, Gastric cancer, P-gp, Rac1

\section{Introduction}

Gastric cancer is the fourth most common type of cancer and the second leading cause of cancer-related deaths in the world [1]. Chemotherapy plays an important role in the treatment of gastric cancer both in adjuvant and advanced settings. However, the efficacy of chemotherapy for gastric cancer is limited due to insensitivity and the development of multi-drug resistance (MDR). Numerous efforts have been made to understand the mechanisms underlying MDR. [2] It is known that MDR involves a large number of molecules and complex mechanisms. Classical drug-resistant molecules, such as P-glycoprotein (P-gp)/ABCB1 and multi-drug resistance protein (MRP1)/ $\mathrm{ABCC} 1$, have been found to play important roles in mediating MDR in some gastric cancers [3]. To reveal the mechanisms underlying drug resistance, we previously compared the proteomic profiles of 5-FU resistant and sensitive colon cancer cells by 2-D gel electrophoresis [4]. We found that RhoGDI2 was up-regulated in 5fluorouracil (5-FU) resistant colon cancer cells (LoVo/

\footnotetext{
* Correspondence: docwuxh@hotmail.com

'Department of Gynecologic Oncology, Fudan University Shanghai Cancer Center, Department of Oncology, Shanghai Medical College, Fudan

University, 270 Dongan Road, Shanghai 200032, People's Republic of China Full list of author information is available at the end of the article
}

5-FU) and that the knockdown of RhoGDI2 expression by transfection with RhoGDI2-specific siRNA significantly increased sensitivity to 5-FU in LoVo/5-FU [4]. These data suggested that RhoGDI2 confers resistance to 5 -FU in colon cancer cells. Later on, our and other groups also showed RhoGDI2 play important role in multi-drug resistance in gastric cancer [5-9].

RhoGDI2 belongs to a family of Rho GTPase dissociate inhibitors (GDIs). GDIs are pivotal regulators of Rho GTPase function typified by forming a complex with Rho GTPase, modulating their nucleotide exchange and membrane association. Therefore, they play an important role in regulating the actin cytoskeleton, cell polarity, microtubule dynamics, membrane transport pathways, and transcription factor activity [10,11]. Unlike other members (RhoGDI1, RhoGDI3), RhoGDI2 is preferentially expressed in hematopoietic cells and overexpressed in gastric cancer it also appears to have a narrow selectivity and lower binding affinity for Rho GTPases [12]. RhoGDI2 associates with and negatively regulates Rac1 and Rac3 in breast cancer cells, but not RhoA, Cdc42, and $\mathrm{RhoC} \mathrm{[13],} \mathrm{whereas} \mathrm{it} \mathrm{positively} \mathrm{regulates} \mathrm{Rac1} \mathrm{in}$ human bladder cancer cells [14]. 
RhoGDI2 is also a substrate for caspases and becomes cleaved in various cell types during apoptosis. Recently, several lines of study reported the important role of RhoGDI2 in multi-drug resistance in gastric cancer [4-9]. A Korea group showed RhoGDI2 confer gastric cancer cells resistance to cisplatin induced apoptosis by

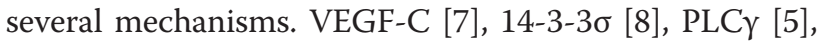
Bcl-2 [6] may mediate the effects of RhoGDI2 in drug resistance. However, our previous study showed a different mechanism [9]. RhoGDI2 reverted the low dose 5FU-induced G2/M arrest rather than high dose 5-FU induced apoptosis [9]. Here we reported another new mechanism by which RhoGDI2 induced MDR, which is that RhoGDI2 upregulates P-gp expression via Rac1.

\section{Results}

\section{Ectopic expression of RhoGDI2 up-regulates P-gp expression}

To determine the role of RhoGDI2 in drug resistance, we established a gastric cancer cell line (MKN-45/RhoGDI2) stably transfected with a RhoGDI2 expression vector. Ectopic expression of RhoGDI2 in MKN-45 increased RhoGDI2 protein and mRNA expression by 58 and 1.6 fold respectively (Figure $1 \mathrm{a}$ and $\mathrm{b}$, both $\mathrm{P}<0.01$ ). The previous study showed ectopic expression of RhoGDI2 confer
MKN-45 resistance to 5-FU. In addition to that, the IC50 of doxorubicin, Paclitaxel was increased in MKN-45 from $0.23 \pm 0.011 \mu \mathrm{M}$ to $0.31 \pm 0.025 \mu \mathrm{M}(P<0.05)$ and from $4.9 \pm 0.33$ to $8.5 \pm 0.60 \mathrm{nM}(P<0.05)$ respectively after ectopic expression of RhoGDI2. To investigate the mechanisms underlying RhoGDI2 induced multi-drug resistance, we compared the mRNA levels of several MDR related genes expression by RT-PCR including Mdr1, multi-drug resistance protein 1 (MRP-1), MRP-2, MRP3, MRP-4, MRP-5, MRP-8 between MKN-45/RhoGDI2 and MKN-45/GFP (Additional file 1 Figure S1). The levels of Mdr-1 (Figure 1b) mRNA was the only one significantly increased in MKN-45/RhoGDI2 by 1.3 fold. Protein level of $M d r-1$ gene (P-gp) was increased by 2.3 fold in MKN45/RhoGDI2, comparing with MKN-45/GFP (Figure 1a, $P<0.05)$. In addition to that, the P-gp activity of MKN$45 /$ RhoGDI2 was also increased by $32.5 \%$, comparing with MKN-45/GFP (Figure 1c, $P<0.05$ ).

\section{RhoGDI2 correlates with P-gp expression in human gastric cancer tissue}

To determine whether there was a correlation between the expression of RhoGDI2 and P-gp in patients with gastric cancer, RhoGDI2 and P-gp levels in gastric cancer tissues were analysed by IHC. As shown in previous
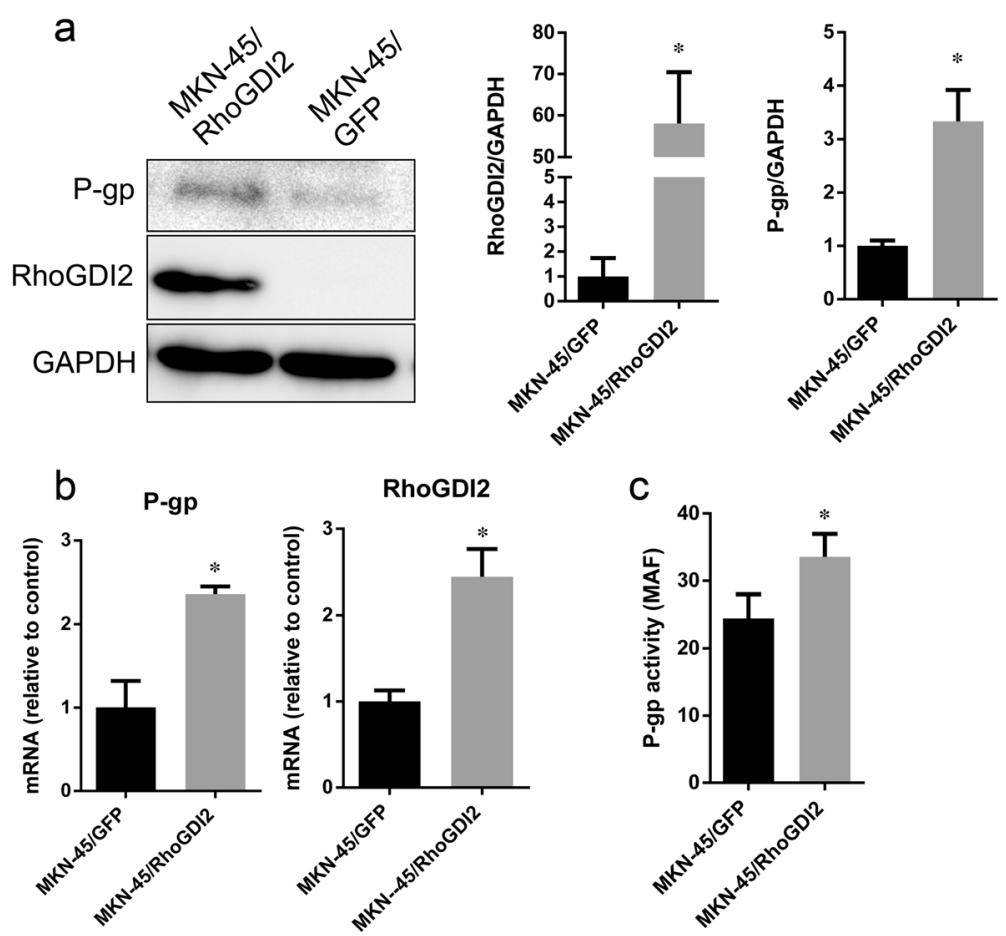

Figure 1 Ectopic expression of RhoGDI2 increased P-gp mRNA, protein and activity. (a) Western blotting analysis of RhoGDI2 and P-gp expression in MKN-45/RhoGDI2 and MKN-45/GFP. The immunoblot image was quantitated by densitometric analysis (right) $(n=3$ independent experiments). ${ }^{*} p<0.05$ vs MKN-45/GFP. (b) The mRNA of RhoGDI2 (left) and P-gp (right) in MKN-45/RhoGDI2 and MKN-45/GFP was detected by RT-PCR. (c) P-gp activity in MKN-45/RhoGDI2 and MKN-45/GFP was measured as described in materials and methods, MAF was plotted. Data are expressed as mean \pm SD from three independent experiments; ${ }^{*} p<0.001$ vs MKN-45/GFP. 
study, RhoGDI2 was overexpressed in gastric cancer cells [9]. P-gp was present in the membrane of both cancerous and adjacent benign gastric epithelial cells. There was no significant difference of the P-gp levels between cancer and benign tissues. Interestingly, the expression of RhoGDI2 was correlated with P-gp expression only in cancerous tissue $\left(p=0.011, \chi^{2}=7.46\right)$. Co-expression of RhoGDI2 and P-gp was also frequently observed in cancer cells (Figure 2).

\section{RhoGDI2 up-regulates P-gp expression via Rac1}

It is reported the RhoGDI2 is associated with Rac1 and regulates its activity [13-17]. Therefore, we tested the Rac1 activity in MKN-45/RhoGDI2 and MKN-45/GFP by ELISA based quantification of active Rac1 (Rac1GTP) level. The Rac1 activity was increased in MKN-45/ RhoGDI2 by 1.6 fold, comparing with MKN-45/GFP (Figure 3a, $P<0.05)$. Next, we test whether Rac1 is required for up-regulation P-gp by RhoGDI2 overexpression. Transfection of Rac1 siRNA for 48 hours decreased Rac1 expression in both MKN-45/RhoGDI2 and MKN45/GFP cells (Figure 3b). P-gp level was also significantly decreased after silencing of Rac1 expression in both MKN-45/RhoGDI2 and MKN-45/GFP cells (Figure 3b). It indicates the effects of RhoGDI2 induced P-gp upregulation was abolished by inhibition of Rac1.

\section{Discussion}

In our previous study using 2D electrophoresis-mass spectrometry, we found that RhoGDI2 was a contributor to 5FU resistance in colon cancer [4]. Later on, we showed that RhoGDI2 also confers resistance to 5 -FU in gastric cancer cells [9]. Here we reported a new mechanism by which RhoGDI2 induces multidrug resistance. It is that RhoGDI2 up-regulates P-gp expression via Rac1.

Accumulative evidence show that RhoGDI2 confer multi-drug resistance in ovarian cancer, gastroenterologic cancer [4-9], breast cancer [13]. It was shown that RhoGDI2 was over-expressed in chemo-resistant fibrosarcoma cells and paclitaxel-resistant ovarian cancers, respectively $[18,19]$. Hee et al. [6] reported that RhoGDI2 confers resistance against multiple chemotherapeutic agents (cisplatin, etoposide, and staurosporin) -induced apoptosis in gastric cancer cells. Together with our results, we conclude that high levels of RhoGDI2 expression are associated with chemotherapy resistance in certain types of cancers, like gastroenterologic cancer.

P-gp, the product of the $A B C B 1$ ( $m d r-1)$ gene, is a full transporter comprised of 12 transmembrane segments divided into TM domains, each linked with an ATPbinding domain [20]. It was first identified due to its overexpression in drug-resistant tumor cells, where it functions as a broad range drug transporter, thereby conferring resistance to many important chemotherapeutic agents including vinblastine, doxorubicin, and paclitaxel $[20,21]$. Here, we reported that ectopic expression of RhoGDI2 increased P-gp expression and activity in gastric cancer. Furthermore, in patients with gastric cancer, the expression of RhoGDI2 was also positively associated with P-gp expression in cancer cells. Our previous study showed RhoGDI2 reverted the low dose 5-FU-induced G2/M arrest. Taken all these together, RhoGDI2 confers multi-drug resistance in gastric cancer by multiple mechanisms. Rho GTPase plays important roles in regulation of astral microtubules and the interaction of spindle microtubules with chromosomes during mitosis. It is reported inhibition of
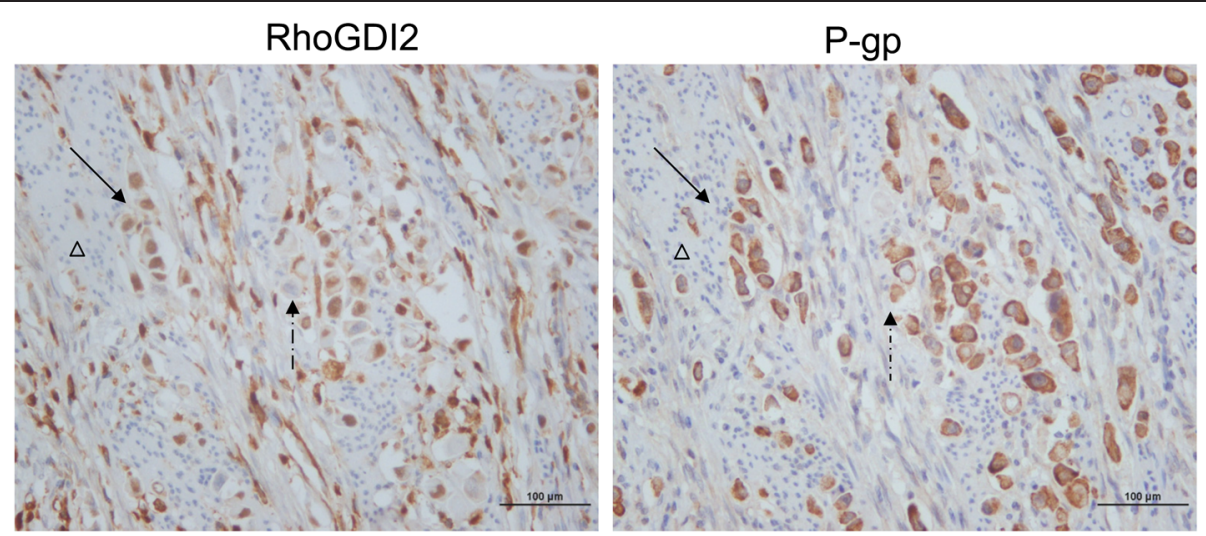

Figure 2 Representative images of IHC staining with anti-P-gp and anti-RhoGDI2 demonstrate the co-expression of P-pg and RhoGDI2 in serial sections of gastric cancer tissue. Serial sectioning slices were stained with anti-P-gp and anti-RhoGDI2 from one case. Brown color represent positive of staining, the negative staining was contra-stained with hematoxylin and shown in blue color. Most of the cancer cells were positively stained with both anti-P-gp and anti-RhoGDI2. RhoGDI2 is expressed majorly in nuclear, whereas P-gp majorly presents in membrane. Some of the cancerous cells expressed high RhoGDI2 also were positively stained with anti-P-gp (shown in solid arrow), whereas some of the cancerous cells with lower RhoGDI2 were also weakly stained with anti-P-gp (shown in dashed arrow). The non-cancerous cells are negative for both P-gp and RhoGDI2 (marked with triangle). Magnification 400x. Scale bar $=100 \mu \mathrm{m}$. 


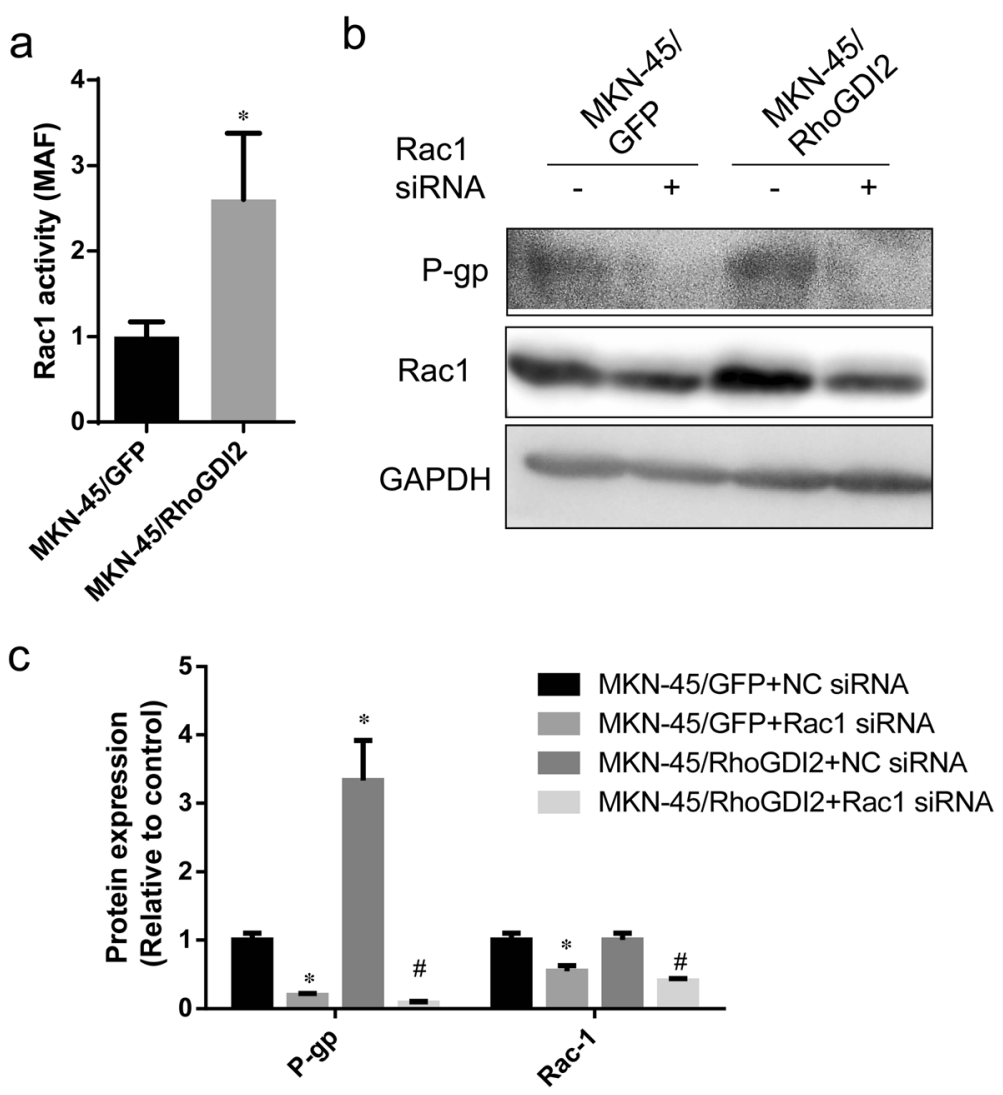

Figure 3 RhoGDI2 up-regulates P-gp expression via Rac1. (a) Rac1 activity in MKN-45/RhoGDI2 and MKN-45/GFP was measured as described in materials and methods. Data are expressed as mean \pm SD from three independent experiments; ${ }^{*} p<0.001$ vs MKN-45/GFP. (b) Western blot analysis of P-gp and Rac1 expression in MKN-45/RhoGDI2, MKN-45/GFP transfected with Rac-1 or Mock siRNA (pictures are representative of three independent experiments). The immunoblot image was quantitated by densitometric analysis (c) $(n=3$ independent experiments). Data was shown in folds relative to MKN-45/GFP + NC siRNA. * $p<0.05$ vs MKN-45/GFP + NC siRNA, \#p<0.05 vs MKN-45/RhoGDI2 + NC siRNA.

Rho GTPase causes a mitotic arrest. Here, we show RhoGDI2 up-regulates P-gp transcription via Rho GTPase (Rac1). Hence it is possible the versatile Rho GTPase contributes to the multiple mechanisms underlining RhoGDI2 induced MDR.

Rac1 was recognized as the important co-operator of RhoGDI2 and mediator of its function [16]. Unlike other member of Rho GTPase, RhoGDI2 preferentially binds to Rac1 and affects its activity [16]. A recent study showed RhoC was also regulated by RhoGDI2 [22]. However, numerous studies have shown the conflicting role of RhoGDI2 in the regulation of Rac1 dependenting on the tumour types and/or cellular microenvironment. it is demonstrated that RhoGDI2 inhibited Rac1 activity in MDAMB-231 human breast cancer cells [13] and mouse embryonic fibroblasts [23], whereas others showed RhoGDI2 acted as a positive regulator of Rac1 in T24 and UMUC3 human bladder cancer cells [14], H9c2 cardiomyoblast cells [17] and ovarian cancer. Both Cho et al. and our results demonstrated that RhoGDI2 up-regulated Rac1 activity [7] in gastric cancer cell lines. The mechanisms by which RhoGDI2 regulates Rac1 aren't fully understood. Boulter et al described an evolutionarily conserved mechanism by which RhoGDI1 controls the homeostasis of Rho proteins in eukaryotic cells [24]. They showed that depletion of RhoGDI1 led to destabilization and degradation of unbound RhoGTPases resulting in a reduction of GTPase protein levels, while unexpectedly activating the remaining membrane bound fraction [24]. It isn't clear whether RhoGDI2 function in a similar manner as RhoGDI1 to protect Rho family GTPases from degradation. Huang et al [17] reported in cardiomyoblast cell line H9c2 unlike RhoGDI1, RhoGDI2 up-regulates Rac1 mRNA transcripts, rather than Rac1 protein stability. Our and other studies didn't show alteration of RhoGDI2 expression could results in Rac1 protein level change in cancer cells $[7,13,14]$. Therefore it is expected RhoGDI2 might act in a different way to regulate Rac1. Extraction Rho GTPases from the membrane by RHOGDIs is the second mechanism to regulated RHO GTPase activity [16,25]. In Breast cancer cells MDA-MB-231, knockdown of RhoGDI2 results in Rac1 translocation from the cytosol 
to cellular membrane compartments, leading to constitutive Rac1 activation and cell growth inhibition [13]. In contrast in bladder cancer, RhoGDI2 activated Rac activity without affecting Rac membrane/cytosol ratios [14]. However, point mutants of RhoGDI2 that increase or decrease the affinity of RhoGDI2 for GTPases abolished its ability to activate Rac [26]. It indicates there is a mechanism distinct from inhibition of membrane association involved in activation of Rac1 by RhoGDI2. Recently, using system biological method, a model was constructed to reveal how RhoGDIs act as positive regulators of Rho GTPase [27]. It indicates RhoGDIs positively regulate Rho GTPase signaling primarily by interacting with GAPs and may participate in the switching between transient and sustained signals of the Rho GTPases. It need further study to confirm whether and which GAPs are involved in our study.

In conclusion, we suggest that there is a new mechanism by which RhoGDI 2 contribute to multi-drug resistance in gastric cancer cells. It may effects drug efflux by up-regulation of P-gp expression via Rac1, rather than disturbance the apoptotic pathway as shown by other group.

\section{Material and methods Cell culture}

Human gastric cancer cell lines MKN-45 were obtained from the Chinese Academy of Sciences Cell Bank of Type Culture Collection (Shanghai, China). All the gastric cancer cell lines were cultured in RPMI-1640 (Invitrogen, Carlsbad, CA) supplemented with 10\% FBS (PAA Laboratories GmbH, Morningside, QLD, Australia).

\section{Patients and tissues}

Human gastric cancer tissue arrays were obtained from Outdo Biotech (Shanghai, China) with 88 individual cases of gastric cancer and matched normal colon tissue. The ethics committee at Ruijin Hospital approved the use of these tissues for research purposes.

\section{RhoGDI2 expressing plasmid}

To generate a RhoGDI2 expression plasmid, the fulllength coding region of RhoGDI2 cDNA was amplified using the primers RhoGDI2-forward CATACTCGAGCGG ACA GAG ACG TGAA GCAC and RhoGDI2-reverse CACTGGATCCGAGT GAC AGG GTG GGA AAAG (the restriction sites of XhoI and BamHI were italic) and inserted into pIRES2-EGFP (Clontech Laboratories, Mountain View, CA) at XhoI and BamHI sites. MKN-45 cells were transfected with pIRES2-EGFP-RhoGDI2 or pIRES2-EGFP using Lipofectamine 2000 reagent (Invitrogen, Carlsbad, CA, USA) according to the manufacturer's instructions. Stable plasmid-transfected clones were selected using $800 \mu \mathrm{g} / \mathrm{ml} \mathrm{G418} \mathrm{(Invitrogen)} \mathrm{for} 2$ weeks, isolated colonies were picked up with tips, and the cells were further cultured in the presence of 400 $\mu \mathrm{g} / \mathrm{ml}$ G418. MKN-45 cells transfected with pIRES2EGFP-RhoGDI2 were named MKN-45/RhoGDI2 cells. MKN-45 cells transfected with pIRES2-EGFP were named MKN-45/GFP cells.

\section{In vitro cytotoxicity assay}

The MTT assay was used to determine the relative sensitivity of cell lines to 5-FU (Xudonghaipu Pharmaceutical Co., Shanghai, China), as described previously [4]. For cell lines, cells plated in 96-well microplates were cultured with growth medium or treated with serial dilutions of 5-FU for 72 hours. Viable cells were measured with MTT (Sigma, St. Louis, MO) and the results were expressed relative to the absorbance of cells grown in the absence of drug. IC50 values were calculated by nonlinear regression analysis from triplicate independent experiments.

\section{Western blot analysis}

Western blot analysis was done as previously described [28]. Briefly, cell lysates were separated by SDS-PAGE and transferred to a PVDF membrane. The blot was then probed with anti-RhoGDI2 (LabVision, Fremont, CA) with a dilution of 1:1000, anti-P-gp (Santa Cruz Biotechnology, Santa Cruz, CA) with a dilution of 1:250, anti-Rac-1 (Sangon Biotech, Shanghai, China ) with a dilution of 1:500, or anti-ABCC-1 (MRP1) (Sangon Biotech, Shanghai, China ) with a dilution of 1:250 followed by an incubation with a horse radish peroxidase-conjugated secondary antibody. The signal was detected using enhanced chemiluminescence (Millipore). The expression level was quantified using Image J program (NIH).

\section{Immunohistochemical staining}

Immunohistochemical staining was done as described previously using a DAKO EnVision + System HRP [29]. RhoGDI2 polyclonal antibody from LabVision (Fremont, CA) was applied at a 1:2,000 dilution overnight at $4^{\circ} \mathrm{C}$, while anti-P-gp was applied at a 1:200 dilution. Purified rabbit-IgG was used as an isotype control. The stained sections were reviewed by two independent observers who had no prior knowledge of the clinic pathologic data of the patients. A scoring method was used as reported previously based on the fact that the specimens clearly showed varying degrees of staining intensity and percentage of cell staining [30]. Briefly, strong-intensity staining was scored as 3 , moderate as 2 , weak as 1 , and negative as 0 . For each intensity score, the percentage of cells with that score was estimated visually. A combined weighted score consisting of the sum of the percentage of cells staining at each intensity level was calculated for each sample. The immunolabelling was categorised as negative (score $>30)$ or positive $($ score $\leq 30)$ for all the tissues. 


\section{P-gp activity (eFluxx-ID Gold uptake assay)}

Fluorescent probe eFluxx-ID Gold was used to monitor P-gp functionality. Cells were trypsinized and incubated for $30 \mathrm{~min}$ at $37^{\circ} \mathrm{C}$ in phenol red free Opti-MEM medium (Invitrogen) with eFluxx-ID Gold (ENZO Life Sciences, Lörrach, Germany) according to the manufacturer's protocol. Cells were analyzed by flow cytometry (FACSCanto; BD Biosciences, Heidelberg, Germany). Each flow cytometry analysis consisted of a record of 100,000 cells. The uptake of eFluxx-ID Gold of EGFP-positive cells was measured in the FL2 (PE) and FL1 (FITC) channel. Dead cells were excluded by using scatter parameters. In parallel experiments, P-gp inhibitor Verapamil was added to the cells to a final concentration of $20 \mu \mathrm{M}$ for 30min during the incubation with eFluxx-ID Gold in order to determine whether the alterations in eFluxx-ID Gold uptake induced by transfection with P-gp. Multidrug resistance activity factor (MAF) was calculated as MAF $($ MDR1 $)=100 \times$ (fluorescence intensity with P-gp inhibitor - fluorescence intensity without P-gp inhibitor)/ fluorescence intensity with P-gp inhibitor.

\section{Rac1 activity assay}

For measurement of Rac1 activation in cell lysates from MKN-45 cells, equal amounts of protein per sample (determined by use of a Protein Assay Kit from Bio-Rad Laboratories; Hercules, CA) were analyzed using the specific Rac1 G-LISA $^{\text {mw }}$ Activation Assay Kit (Cytoskeleton; Denver, CO) according to the manufacturer's instructions. This luminescence G-LISA ${ }^{\text {Tx }}$ is an ELISA based assay that allows measuring the GTP-bound (active) form of small G-proteins. Active Rac1 levels were expressed as fold-increase over the active Rac1 levels in control conditions.

\section{Reverse Transcription-Polymerase Chain Reaction (RT-PCR) for the Detection of P-gp RhoGDI2 Expression at the mRNA Level}

Expression of human P-gp and RhoGDI2 at the mRNA level in the cell lines used was verified by reverse transcription of RNA followed by polymerase chain reaction. RNA was isolated using the Trizol reagent (Invitrogen Life Technologies, Carlsbad, CA, USA). First-strand cDNA synthesis was performed with the First-Strand cDNA synthesis kit for RT-PCR (Jrdun biotechnology, Shanghai, China) with oligo(d T) primers according to the manufacturer's instructions. Primers used for the amplification of human P-gp were 5' - CGCTGTTCGTTTCCTTTAG -3' (sense) and 5' - CTTCTTTGCTCCTCCATTG -3' (antisense). For RhoGDI2, primers were 5'-TTCTTCACCGA CGATGAC-3' (sense) and 5'-GGAAATGTG GCAGT GTTG-3' (antisense). For MRPs, see Additional file 2 Table S1. All primers were synthesized by MWG Biotech AG (Shanghai, China). PCR was performed on the ABI
Prism 7300 in a total volume of $50 \mu \mathrm{l}$ using the Jrdun biotechnology FQ-PCR kit (Shanghai, China).

\section{Statistical analysis}

Statistical analyses of data were performed by using the Student t-test or one-way analysis of variance, depending on the number of groups in comparison. Data that failed the test for normal distribution or homogeneous variance were analysed using the Mann-Whitney $U$ or the Kruskal-Wallis tests. The correlation between RhoGDI2 and P-gp expression was analysed using the Fisher exact test. The statistical software SPSS version 14.0 was used for analysis. Significance was set at $\mathrm{P}<0.05$.

\section{Additional files}

Additional file 1: Figure S1. A. The mRNA of RhoGDI2 (left) and P-gp (right) in MKN-45/RhoGDI2 and MKN-45/GFP was detected by RT-PCR. Data was expressed as relative to GAPDH (mean \pm SD) from three independent experiments; ${ }^{*} p<0.05$ vs MNK-45/GFP. B. Western blotting analysis of MRP-1 expression in MKN-45/RhoGDI2 and MKN-45/GFP.

Additional file 2: Table S1. The primers for MRPS.

\section{Abbreviations}

MDR: Multidrug resistance; GDls: Rho GTPase dissociate inhibitors; P-gp: P-glycoprotein; MRP: Multi-drug resistance protein; 5-FU: 5-fluorouracil.

\section{Competing interests}

The authors declare that they have no competing interests.

\section{Authors' contributions}

ZZ designed and performed the experiments, analyzed the data, and wrote the manuscript. LBY helped with designing the experiments, helped with performing the $\mathrm{IHC}$ experiments, analyzing the data, and evaluating the manuscript. WXH designed the experiments, analyzed the data, and revised the paper. ZZ carried out the molecular genetic studies, participated in the sequence alignment and drafted the manuscript. All authors read and approved the final manuscript.

\section{Acknowledgments}

This work was supported by National Natural Science Foundation of China (No.81101649).

\section{Author details}

${ }^{1}$ Department of Gynecologic Oncology, Fudan University Shanghai Cancer Center, Department of Oncology, Shanghai Medical College, Fudan University, 270 Dongan Road, Shanghai 200032, People's Republic of China. ${ }^{2}$ Department of Surgery, Shanghai Key Laboratory of Gastric Neoplasms, Shanghai Institute of Digestive Surgery, and Gastroenterology, Ruijin Hospital, Jiaotong University School of Medicine, Shanghai, China.

Received: 10 December 2014 Accepted: 28 March 2015

Published online: 15 April 2015

\section{References}

1. Jemal A, Bray F, Center MM, Ferlay J, Ward E, Forman D. Global cancer statistics. CA Cancer J Clin. 2011;61(2):69-90.

2. Longley DB, Harkin DP, Johnston PG. 5-fluorouracil: mechanisms of action and clinical strategies. Nat Rev Cancer. 2003;3(5):330-8.

3. Zhang D, Fan D. New insights into the mechanisms of gastric cancer multidrug resistance and future perspectives. Future Oncol. 2010;6(4):527-37.

4. Zheng Z, Li J, He X, Chen X, Yu B, Ji J, et al. Involvement of RhoGDI2 in the resistance of colon cancer cells to 5 -fluorouracil. Hepatogastroenterology. 2010;57(102-103):1106-12. 
5. Cho HJ, Baek KE, Nam IK, Park SM, Kim IK, Park SH, et al. PLCgamma is required for RhoGDI2-mediated cisplatin resistance in gastric cancer. Biochem Biophys Res Commun. 2011;414(3):575-80.

6. Cho HJ, Baek KE, Park SM, Kim IK, Nam IK, Choi YL, et al. RhoGDI2 confers gastric cancer cells resistance against cisplatin-induced apoptosis by upregulation of Bcl-2 expression. Cancer Lett. 2011;311(1):48-56.

7. Cho HJ, Kim IK, Park SM, Baek KE, Nam IK, Park SH, et al. VEGF-C mediates RhoGDI2-induced gastric cancer cell metastasis and cisplatin resistance. Int J Cancer. 2014;135(7):1553-63.

8. Kim IK, Park SM, Cho HJ, Baek KE, Nam IK, Park SH, et al. 14-3-3sigma attenuates RhoGDI2-induced cisplatin resistance through activation of Erk and p38 in gastric cancer cells. Oncotarget. 2013;4(11):2045-56.

9. Zheng Z, He XY, Li JF, Yu BQ, Chen XH, Ji J, et al. RhoGDI2 confers resistance to 5 -fluorouracil in human gastric cancer cells. Oncology Letters. 2013;5(1):255-60.

10. Heasman SJ, Ridley AJ. Mammalian Rho GTPases: new insights into their functions from in vivo studies. Nat Rev Mol Cell Biol. 2008;9(9):690-701.

11. Jaffe $A B$, Hall A. Rho GTPases: biochemistry and biology. Annu Rev Cell Dev Biol. 2005;21:247-69.

12. Dovas A, Couchman JR. RhoGDI: multiple functions in the regulation of Rho family GTPase activities. Biochem J. 2005;390(Pt 1):1-9.

13. Zhang Y, Rivera Rosado LA, Moon SY, Zhang B. Silencing of D4-GDI inhibits growth and invasive behavior in MDA-MB-231 cells by activation of Rac-dependent p38 and JNK signaling. J Biol Chem. 2009;284(19):12956-65.

14. Moissoglu K, McRoberts KS, Meier JA, Theodorescu D, Schwartz MA. Rho GDP dissociation inhibitor 2 suppresses metastasis via unconventional regulation of RhoGTPases. Cancer Res. 2009;69(7):2838-44.

15. Agarwal NK, Chen CH, Cho H, Boulbes DR, Spooner E, Sarbassov DD. Rictor regulates cell migration by suppressing RhoGDI2. Oncogene. 2013;32(20):2521-6.

16. Griner EM, Theodorescu D. The faces and friends of RhoGDI2. Cancer Metastasis Rev. 2012;31(3-4):519-28.

17. Huang CY, Yang LC, Liu KY, Chang IC, Liao PH, Chou Jl, et al. ZAK negatively regulates RhoGDlbeta-induced Rac1-mediated hypertrophic growth and cell migration. J Biomed Sci. 2009;16:56.

18. Sinha P, Hutter G, Kottgen E, Dietel M, Schadendorf D, Lage H. Search for novel proteins involved in the development of chemoresistance in colorectal cancer and fibrosarcoma cells in vitro using two-dimensional electrophoresis, mass spectrometry and microsequencing. Electrophoresis. 1999;20(14):2961-9.

19. Goto T, Takano M, Sakamoto M, Kondo A, Hirata J, Kita T, et al. Gene expression profiles with CDNA microarray reveal RhoGDI as a predictive marker for paclitaxel resistance in ovarian cancers. Oncol Rep. 2006;15(5):1265-71.

20. Borst $P$, Elferink RO. Mammalian ABC transporters in health and disease. Annu Rev Biochem. 2002;71:537-92.

21. Leonard GD, Fojo T, Bates SE. The role of ABC transporters in clinical practice. Oncologist. 2003;8(5):411-24.

22. Griner EM, Dancik GM, Costello JC, Owens C, Guin S, Edwards MG, et al. RhoC Is an Unexpected Target of RhoGDI2 in Prevention of Lung Colonization of Bladder Cancer. Mol Cancer Res. 2015;13(3):483-92.

23. Agarwal NK, Kazyken D, dos Sarbassov D. Rictor encounters RhoGDI2: the second pilot is taking a lead. Small GTPases. 2013;4(2):102-5.

24. Boulter E, Garcia-Mata R, Guilluy C, Dubash A, Rossi G, Brennwald PJ, et al. Regulation of Rho GTPase crosstalk, degradation and activity by RhoGDI1. Nat Cell Biol. 2010;12(5):477-83.

25. Garcia-Mata R, Boulter E, Burridge K. The 'invisible hand': regulation of RHO GTPases by RHOGDIs. Nat Rev Mol Cell Biol. 2011;12(8):493-504.

26. Wu Y, Moissoglu K, Wang H, Wang X, Frierson HF, Schwartz MA, et al. Src phosphorylation of RhoGDI2 regulates its metastasis suppressor function. Proc Natl Acad Sci U S A. 2009;106(14):5807-12.

27. Ota T, Maeda M, Okamoto M, Tatsuka M. Positive regulation of Rho GTPase activity by RhoGDIs as a result of their direct interaction with GAPS. BMC Syst Biol. 2015;9:3.

28. Wang YW, Qu Y, Li JF, Chen XH, Liu BY, Gu QL, et al. In vitro and in vivo evidence of metallopanstimulin-1 in gastric cancer progression and tumorigenicity. Clin Cancer Res. 2006;12(16):4965-73.
29. Yuen HF, Chan YP, Chan KK, Chu YY, Wong ML, Law SY, et al. Id-1 and Id-2 are markers for metastasis and prognosis in oesophageal squamous cell carcinoma. Br J Cancer. 2007;97(10):1409-15.

30. Ben QW, Wang JC, Liu J, Zhu Y, Yuan F, Yao WY, et al. Positive expression of L1-CAM is associated with perineural invasion and poor outcome in pancreatic ductal adenocarcinoma. Ann Surg Oncol. 2010;17(8):2213-21.

\section{Submit your next manuscript to BioMed Central and take full advantage of:}

- Convenient online submission

- Thorough peer review

- No space constraints or color figure charges

- Immediate publication on acceptance

- Inclusion in PubMed, CAS, Scopus and Google Scholar

- Research which is freely available for redistribution 